\title{
Article \\ Microstructure Characterization and Small Punch Test Analysis in Nickel-Based Alloy 617 by High Energy Neon Implantation
}

\author{
Yuanhua Shen ${ }^{1,2}$, Shasha $\mathrm{Lv}^{3, *}$, Qin Zhou ${ }^{4, *}, \mathrm{Li} \mathrm{Shi}^{4}{ }^{4}$ Libin Sun ${ }^{4}$ and Zhengcao $\mathrm{Li}^{1}$ \\ 1 Key Lab of Advanced Materials (MOE), School of Materials Science and Engineering, Tsinghua University, \\ Beijing 100084, China; yh-shen21@mails.tsinghua.edu.cn (Y.S.); zcli@tsinghua.edu.cn (Z.L.) \\ 2 Department of Engineering Physics, Tsinghua University, Beijing 100084, China \\ 3 Key Laboratory of Beam Technology, Ministry of Education, College of Nuclear Science and Technology, \\ Beijing Normal University, Beijing 100875, China \\ 4 Institute of Nuclear and New Energy Technology, Tsinghua University, Beijing 100084, China; \\ shili@tsinghua.edu.cn (L.S.); slb@tsinghua.edu.cn (L.S.) \\ * Correspondence: lvss@bnu.edu.cn (S.L.); qinzhou@tsinghua.edu.cn (Q.Z.)
}

check for updates

Citation: Shen, Y.; Lv, S.; Zhou, Q.; Shi, L.; Sun, L.; Li, Z. Microstructure Characterization and Small Punch Test Analysis in Nickel-Based Alloy 617 by High Energy Neon Implantation. Metals 2022, 12, 438. https://doi.org/10.3390/ met12030438

Academic Editor: Liping Guo and Ian Jones

Received: 30 November 2021 Accepted: 28 January 2022

Published: 2 March 2022

Publisher's Note: MDPI stays neutral with regard to jurisdictional claims in published maps and institutional affiliations.

Copyright: (C) 2022 by the authors. Licensee MDPI, Basel, Switzerland. This article is an open access article distributed under the terms and conditions of the Creative Commons Attribution (CC BY) license (https:// creativecommons.org/licenses/by/ $4.0 /)$.

\begin{abstract}
Nickel-based alloys are good candidate structural materials for ultra-high temperature gascooled reactors due to their excellent mechanical properties under high-temperature conditions. The operating environment in the compact high-temperature gas-cooled reactor proposes more stringent requirements with higher displacement and temperatures higher than $700{ }^{\circ} \mathrm{C}$. The irradiation resistance of nickel-based alloy 617 is evaluated by $120 \mathrm{MeV}$ neon ion irradiation. The neon ion fluence is set with reference to the number of helium atoms generated by the reaction of $(n, \alpha)$ in nickel-based alloy under actual service conditions. With the application of an energy gradient degrader, the irradiation damage area is more uniform with increasing depth. The neon ion irradiation causes no significant surface damage to alloy 617 while the evolution of defects such as inherent dislocation lines is affected, thus leading to the deterioration of mechanical properties. By using the small punch test to analyze the irradiation effects, it is found that the neon ion irradiation results in the tendency of irradiation softening and does not lead to changes in the basic fracture characteristics under different annealing conditions. After annealing at 700 and $800{ }^{\circ} \mathrm{C}$ for $2 \mathrm{~h}$, the irradiation embrittlement trend is intensified, with the fracture characteristic values of irradiated samples showing a more obvious decreasing trend.
\end{abstract}

Keywords: nickel-based alloy; neon ion irradiation; microstructure; mechanical properties

\section{Introduction}

Nuclear energy is low-carbon and clean, and the ongoing research of fourth-generation nuclear power technology is expected to ensure safe and efficient use [1]. The operating environment of the fourth-generation reactor system is more stringent [2]. However, traditional structural materials struggle to meet the corresponding requirements. Therefore, the search for new reactor structural materials has become a key issue in the development of fourth-generation nuclear power technology. Nickel-based alloys have excellent mechanical properties at high temperatures and can be used as candidate materials for the fourthgeneration reactor system. Among them, alloy 617 is a solution strengthened nickel-base alloy, mainly composed of nickel, chromium, cobalt and molybdenum.

The Idaho National Laboratory (INL) and other research institutes in the United States have tested the performance of alloy 617, and finally, they found that alloy 617 has excellent performance at high temperature and can be used in the fourth-generation reactor with a working temperature of more than $760{ }^{\circ} \mathrm{C}$ [3-5]. In addition to the high-temperature environment, the high neutron irradiation dose in the reactor can also affect the use of structural materials, so the irradiation resistance also needs to be concerned. In order to 
explore the irradiation resistance of nickel-base alloys, relevant research is conducted from two aspects of microstructure and mechanical properties.

The research on microstructure can be roughly divided into: (1) The microstructural changes under different conditions. The influences of external factors such as irradiation dose, irradiation dose rate, ion energy and irradiation temperature on the irradiationinduced defects are explored. Furthermore, the influences of internal factors such as solute atoms on the microstructure are explored. (2) Interaction of defects [6]. Jin et al. observed the microstructure changes of $\mathrm{Ar}$ and $\mathrm{Ni}$ irradiated nickel-based alloy C-276 by transmission electron microscopy (TEM). The results showed that microscopic defects such as the dislocation were observed in the irradiated samples. With the rise of irradiation temperature and dose, the size of irradiation-induced defects increased, while its density showed an opposite trend and decreased $[7,8]$. Chen explored the effect of annealing temperature on irradiated defects of nickel-based alloy by an in-situ annealing experiment by Xe ions. The results showed that the density of irradiation-induced defects did not change a lot when the annealing temperature is lower than $600{ }^{\circ} \mathrm{C}$. When the annealing temperature is higher than $650{ }^{\circ} \mathrm{C}$, the defect density decreased sharply. It is analyzed that the reduction in defect density is related to the recombination of interstitial dislocations or cluster atoms with vacancies [6]. Kojima et al. studied nickel and nickel-based alloy irradiated by neutrons and found that solute atoms of very large or very small size contributed to the formation of dislocation loops in the matrix, while the atoms of moderate size promoted the formation of dislocation loops near the dislocations [9]. In addition, irradiation-induced defects can interact with defects such as inherent dislocations in the alloy, thus affecting the microstructure of the alloy. After irradiating Hastelloy $\mathrm{N}$ alloy with Xe ions, the irradiation-induced defects tended to cluster near the inherent dislocations and modified the dislocations at a low damage dose. The number density of the inherent dislocations decreased with the increase in the irradiation damage dose. This study attributed the disappearance of the inherent dislocations to the climbing of the dislocations towards the free surface by absorbing the interstitials [10].

After irradiation, the mechanical properties of nickel-based alloys such as the hardness and toughness will change. The effect of ion irradiation on alloy hardness can be divided into two categories, namely irradiation hardening and irradiation softening.

A nickel-based alloy (Ni-17Mo-7Cr) was irradiated with Xe ions, and the hardness of the alloy was measured using a nano-indentation instrument by Huang et al. The results showed that compared with the unirradiated sample, the hardness of the irradiated sample showed a significant increase with the increase in the irradiation damage dose [11,12]. Another study showed that the hardness of the irradiated samples fluctuated slightly and did not change significantly at a higher damage dose, indicating the occurrence of irradiation hardening saturation [13]. Liu explored the effect of helium ion irradiation on the hardness of a nickel-base alloy GH3535. The results showed that the hardness increment of the irradiated samples decreased with high-temperature annealing under the same conditions [14]. Based on the theory of the pinning effect, Zhang et al. thought that irradiation-induced defects would hinder the movement of dislocation lines. Combined with the Orowan equation and experimental data, they found that irradiation hardening was correlated with changes in the size and density of microscopic defects [15].

Less research has been undertaken on irradiation softening than on the irradiation hardening phenomenon. After irradiation with $3 \mathrm{MeV} \mathrm{Au}$ ions, Guo et al. found that at lower doses (0-10 dpa) the measured nanohardness values for the Ni-17Mo-7Cr alloy increased with increased irradiation damage dose and at higher doses (10-30 dpa) the irradiation softening effect dominated [16]. Changizian et al. investigated the effects of nickel ions irradiation on X-750 alloys at room temperature and found the softened phenomenon compared to unirradiated materials. A similar irradiation softening phenomenon was found in the Inconel 718 alloy [17].

Irradiation embrittlement can affect the actual effect of nickel-based alloys, which is studied by researchers. The ductility of Hastelloy $\mathrm{N}$ alloys after neutron irradiation was 
tested by Martin et al. It is shown that the irradiation makes the ductility performance of the alloy decrease, and high temperatures can aggravate this brittle effect [18]. From 1956 to 1975, several nickel-based alloys (Alloy 600, 625, 706, 718, 800, Hastelloy X, A-286 and Rene 41) were irradiated under high temperatures in material test reactors. The results of tensile tests showed that irradiation resulted in a significant reduction in total elongation of all alloys, with grain boundary helium attributed to the main factor of ductility loss [19].

The related database of irradiation performance of the nickel-based alloys has not been well established and the research on the irradiation effects are not enough for a proper evaluation of their irradiation resistance. In this paper, the nickel-based alloy 617 is irradiated by $\mathrm{Ne}$ ions with a maximum energy of $120 \mathrm{MeV}$ to analyze the irradiation effects. In terms of microstructure characterization, scanning electron microscopy (SEM) is used to explore the effect of irradiation on the surface morphology. Transmission electron microscopy (TEM) and positron annihilation spectrum (PAS) are used to explore the impact of irradiation on the internal defects of alloy 617. The small punch test (SPT) is used to analyze the irradiation effects on the tensile strength and fracture properties of alloy 617 before and after high temperature annealing.

\section{Methods}

\subsection{Materials and Ion Irradiation Experiment}

The nickel-based alloy 617 was used, with the main components shown in Table 1. According to the instrument requirements of the characterization experiment, the original samples were cut into sheet samples of size $10 \mathrm{~mm} \times 10 \mathrm{~mm} \times 1 \mathrm{~mm}$. To meet the experimental requirements for sample surface roughness, the cut sheet samples were sanded with sandpaper of 200 \#, 600 \#, 1000 \#, 1500 \#, 2000 \#, 3000 \#. Next, the samples were polished with silica polishing solution (granularity of $0.02 \mu \mathrm{m}$ ). Finally, the samples were sonicated with acetone for $30 \mathrm{~min}$.

Table 1. The main chemical composition of alloy 617.

\begin{tabular}{ccccccccc}
\hline Elements & Ni & Cr & Co & Mo & Fe & Mn & Si & Al \\
\hline wt. $\%$ & $\geq 44.5$ & $20.0 \sim 24.0$ & $10 \sim 15$ & $8.0 \sim 10.0$ & $\leq 3$ & 1.00 & 1.00 & $0.8 \sim 1.5$ \\
\hline
\end{tabular}

Irradiation experiments were completed at the Heavy Ion Accelerator Laboratory (HIRFL) of the Lanzhou Institute of Modern Physics. Only one surface of the samples was irradiated by $\mathrm{Ne}$ ions whose maximum energy was $120 \mathrm{MeV}$, and the irradiation area was $10 \mathrm{~mm} \times 10 \mathrm{~mm}$. The migration, aggregation behavior of Ne ions in metals is similar to He. Meanwhile, Ne also has a larger atomic displacement cross-section, which enables it to introduce high displacement damage in the alloy. Thus, high-energy neon ion irradiation can be used as an alternative to helium ion irradiation [20]. The temperature of the samples increased during high-energy ion implantation. Referring to a similar study, the temperature during the irradiation experiment is estimated to be around $440{ }^{\circ} \mathrm{C}$ [20].

In addition, in order to make the experimental conditions as close as the actual service conditions, the amount of helium generated by nuclear transmutation was calculated by using the neutron flux in the actual reactor. Taking it as a reference value, the equal number of neon ions as well as 10 times more neon ions were implanted, respectively, to analyze the helium brittle problem in the reactor. In addition, an energy gradient degrader device was used for some samples to obtain five kinds of incident neon ions with different energies, thus making the irradiation damage distribution more even.

The SRIM2013 procedure was used to simulate the irradiation damage distribution inside the alloy 617. The calculation mode was "ion distribution and quick calculation of damage", the displacement threshold energy selected to $40 \mathrm{eV}$, lattice binding energy set to $0 \mathrm{eV}$. The simulation results are shown in Figure 1, and the detailed data for the ion-irradiated samples are shown in Table 2. 

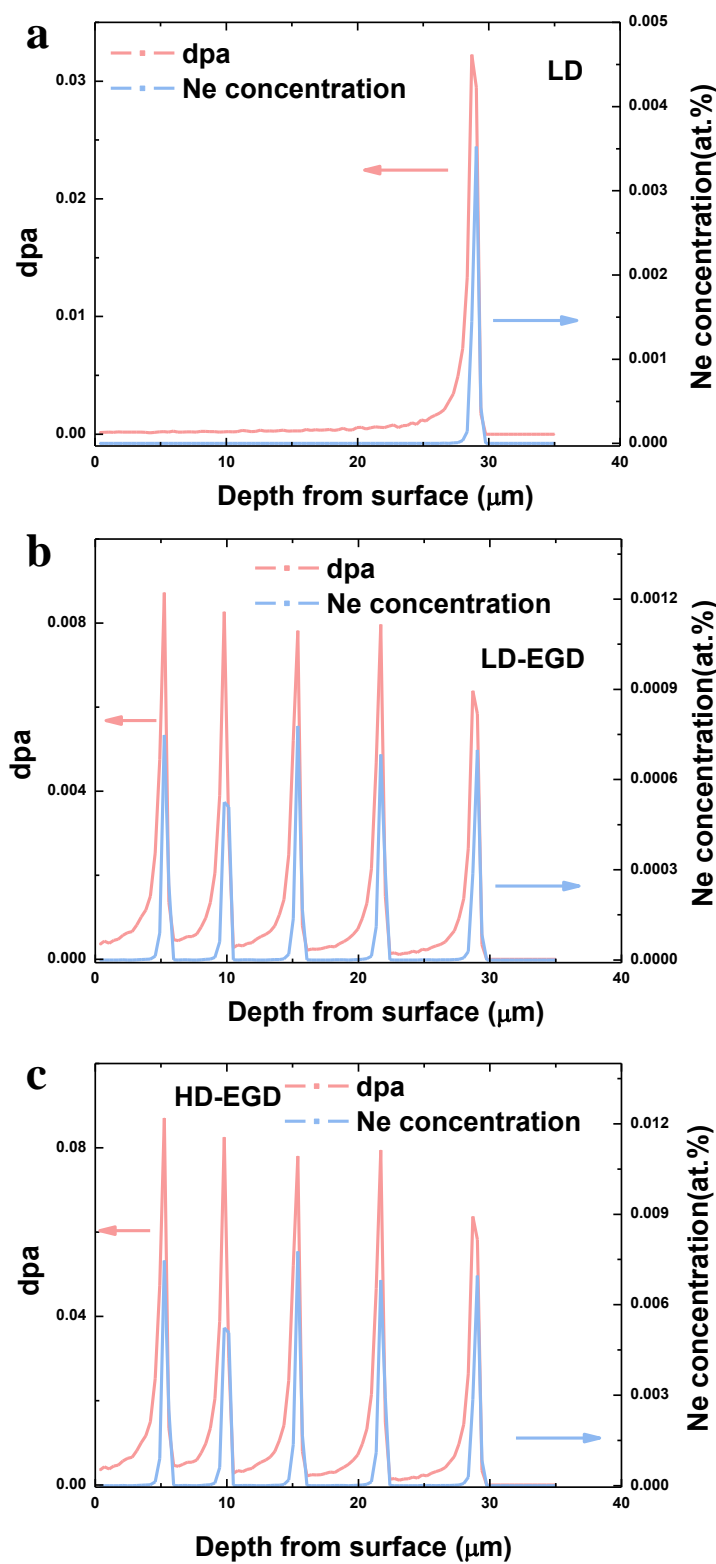

Figure 1. SRIM simulation of neon ion concentration distribution and damage distribution: (a) low dose (LD), (b) low dose with energy gradient degrader (LD-EGD), (c) high dose with energy gradient degrader (HD-EGD).

Table 2. Detailed data of ion-irradiation experiments.

\begin{tabular}{|c|c|c|c|c|}
\hline Ion Energy (MeV) & Sample & $\begin{array}{l}\text { Ion Fluence } \\
\left(\text { ions } / \mathrm{cm}^{2}\right)\end{array}$ & $\begin{array}{l}\text { Irradiation Damage } \\
\qquad\left(\text { dpa }^{1}\right)\end{array}$ & Other Information \\
\hline 120 & Low dose (LD) & $1.67 \times 10^{14}$ & 0.0322 & $\begin{array}{c}\text { without energy gradient } \\
\text { degrader }\end{array}$ \\
\hline $24,48,72,96,120$ & $\begin{array}{l}\text { Low dose with energy } \\
\text { gradient degrader } \\
\text { (LD-EGD) }\end{array}$ & $1.66 \times 10^{14}$ & 0.0078 & $\begin{array}{c}\text { with energy gradient } \\
\text { degrader } \\
\text { (speed: } 0.1 \mathrm{r} / \mathrm{s} \text { ) }\end{array}$ \\
\hline $24,48,72,96,120$ & $\begin{array}{l}\text { High dose with energy } \\
\text { gradient degrader } \\
\text { (HD-EGD) }\end{array}$ & $1.65 \times 10^{15}$ & 0.0781 & $\begin{array}{c}\text { with energy gradient } \\
\text { degrader } \\
\text { (speed: } 0.1 \mathrm{r} / \mathrm{s} \text { ) }\end{array}$ \\
\hline
\end{tabular}

${ }^{1}$ displacement per atom. 


\subsection{Microstructure Characterization}

The surface morphology was observed by a ZEISS field emission scanning electron microscope, and its main parameters are as follows: acceleration voltage $(0.05-30 \mathrm{kV})$, detector (SE2, InlensDuo, BSE), resolution: $(1.0 \mathrm{~nm} / 15 \mathrm{kV}, 1.7 \mathrm{~nm} / 1 \mathrm{kV})$, energy spectrum detection range (B4-U92). The characterization of transmission electron microscopy (TEM) requires electron beams to pass through the sample, and the thickness of the sample generally does not exceed $100 \mathrm{~nm}$, so further sample preparation work is required. The TEM samples in this paper are prepared using a focused ion beam (FIB) technique. The FIB system using Ga ion beams is produced by Carl Zeiss in Germany, whose instrument model is Crossbeam 340. Microstructure examination was performed using JEOL-2100 TEM.

\subsection{Small Punch Test}

For the small punch test, additional sample preparation work is also required. Taking into account the main irradiation damage distribution depth range $(0-30 \mu \mathrm{m})$ and sample requirements for the small punch test, the sheet samples $(10 \mathrm{~mm} \times 10 \mathrm{~mm} \times 1 \mathrm{~mm})$ were thinned to about $80 \mu \mathrm{m}$ by grinding, and their surfaces were washed with alcohol. Subsequently, a punching machine with a pore diameter of $\Phi 3 \mathrm{~mm}$ was used to stamp the sheet samples into small discs of $\Phi 3 \mathrm{~mm}$. Then, the sample thickness was measured. It is finally loaded into a test device for testing, the test temperature is room temperature, and the stamping speed of the small punch is $0.2 \mathrm{~mm} / \mathrm{min}$. The device schematic of the small punch test is shown in Figure S1, in which the punch and the ball are $1 \mathrm{~mm}$ in diameter [21].

Due to the complex stress state in the small punch test, it is difficult to use the original data directly, so further processing of the original data is required. The two commonly used methods are numerical simulation and the empirical correlation method. In this paper, the second method is used to correlate the characteristic data on the original load-deflection curves with the conventional test results to evaluate the changes in the tensile properties and fracture properties [22,23].

The ultimate tensile strength can reflect the changes in the tensile properties of the material. Studies have shown that the ultimate tensile strength can be estimated from two characteristic data, which are $P_{\max }$ the maximum load, and $P_{i}$ the load corresponding to the deflection of 1.1 times sample thickness [22,23]. The corresponding empirical correlations have been derived to analyze the irradiation effect caused by ion and neutron irradiation for some specific materials [20]. The applicability of these correlations has been explored by some researchers. It shows that a unified empirical correlation can be used to roughly estimate the ultimate tensile strength under some different test conditions [23,24]. Therefore, it can be used to estimate ultimate tensile strength with these characteristic data, though there are no corresponding correlations for nickel-based alloys in the literature. The tensile characteristic values $\left(\frac{P_{\max }}{u_{m} t_{0}}, \frac{P_{i}}{t_{0}^{2}}\right)$ have a linear proportional relationship with the ultimate tensile strength, which is the basis of the following analysis. Altstadt et al. found that $P_{i}$ may be more universally appropriate for estimating the ultimate tensile strength for a range of materials, as it can indicate the onset of necking or plastic instability within the SP sample and the correlation constant for it is more independent of material properties [24]. Therefore, the tensile characteristic values $\frac{P_{i}}{t_{0}^{2}}$ are used to estimate ultimate tensile strength in this paper.

Fracture toughness can reflect the changes in the fracture properties, and it is also a parameter that characterizes the ability of the sample to prevent crack propagation. Fracture strain can be used to estimate changes in fracture toughness, for they are inherently correlated. In the current study, the equivalent fracture strain is used to estimate the fracture toughness of ductile materials, and the equivalent fracture strain can be calculated with the fracture deflection $u_{f}$. Regarding the relationship between fracture deflection and fracture toughness, there is a series of empirical correlations. Although the correlation constants depend on the different materials, the basic form of the empirical correlations has not changed, from which the positive correlation between the fracture characteristic value $\frac{u_{f}}{t_{0}}$ 
and fracture toughness can be found [22,23]. Currently, the definition of fracture deflection $u_{f}$ has not been unified. There are several definitions of $u_{f}$, which are the deflection at the maximum force, the deflection where the force has dropped by $20 \%$ after reaching its maximum, and the deflection where the force has dropped by $30 \%$ after reaching its maximum $[20,25]$. In this paper, the fracture deflection is considered to correspond to the $20 \%$ load drop with respect to the maximum load $P_{\max }$ with the European standard CWA15627 and ASTM E3205-20 standard for reference [26,27].

\section{Results and Discussions}

\subsection{Microstructure Characterization}

\subsubsection{The Surface Morphology by SEM}

To analyze the surface damage of alloy 617 caused by high-energy neon ion irradiation, the surface morphology is observed with SEM. The relevant results are shown in Figure 2. No significant difference in the surface morphology is observed between irradiated and unirradiated samples.

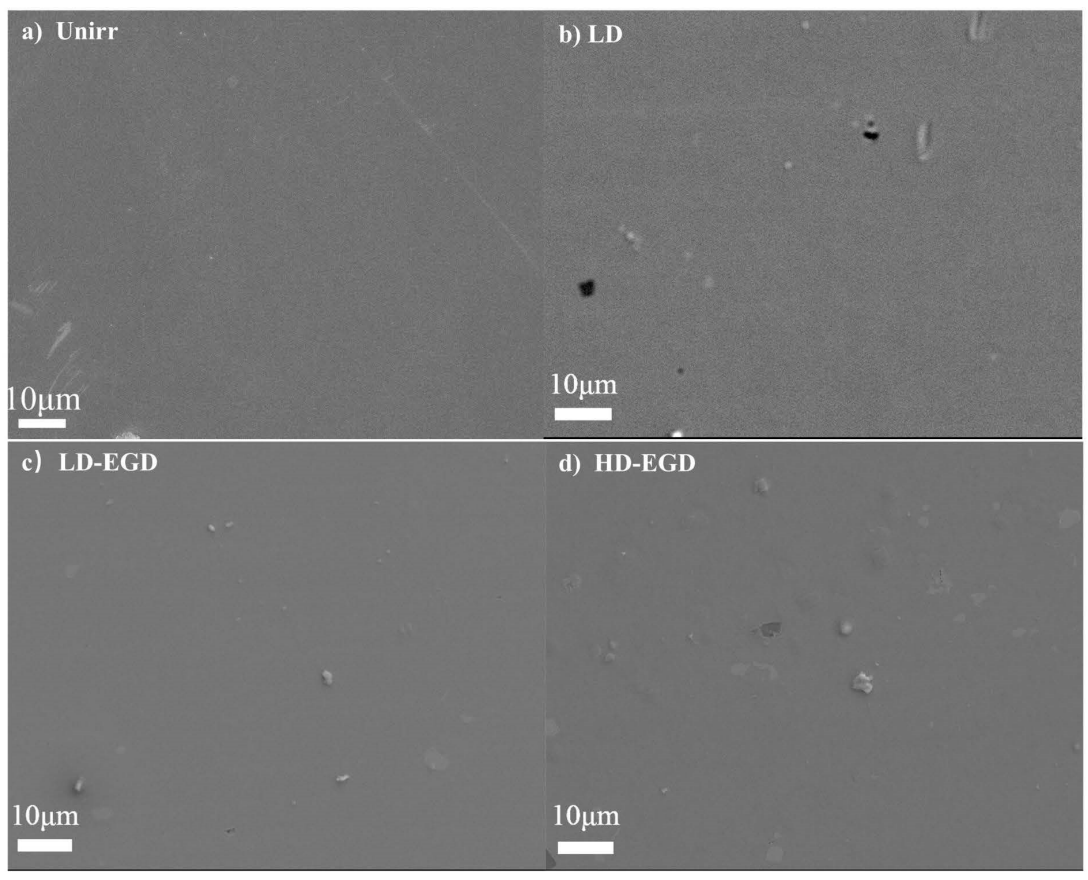

Figure 2. Surface morphology observations of different irradiated samples: (a) unirradiated sample, (b) low dose (LD), (c) low dose with energy gradient degrader (LD-EGD), (d) high dose with energy gradient degrader (HD-EGD).

\subsubsection{TEM Analysis on Defects}

The TEM results are shown in Figure 3. In the unirradiated sample, there are a large number of inherent dislocation lines and obvious grain boundaries. In the irradiated samples (LD-EGD, HD-EGD), not only the existence of inherent dislocation lines and grain boundaries but also a large number of stacking faults and a small number of dislocation loops are observed. In addition, neon bubbles are not obviously observed in the irradiated samples.

\subsubsection{Positron Annihilation Analysis}

The original life spectrum is performed by the three-state fitting to obtain the value of the three life components and their annihilation intensity, as shown in Figure 4. Furthermore, the average lifetime value is calculated. For each sample, we repeat the experiment twice. It can be found that the lifetime value of the first life component is between 142 and $147 \mathrm{ps}$, the lifetime value of the second life component is between 233 and 259 ps, and the 
third life component is only found in the irradiated sample HD-EGD, whose lifetime value is above $2000 \mathrm{ps}$.

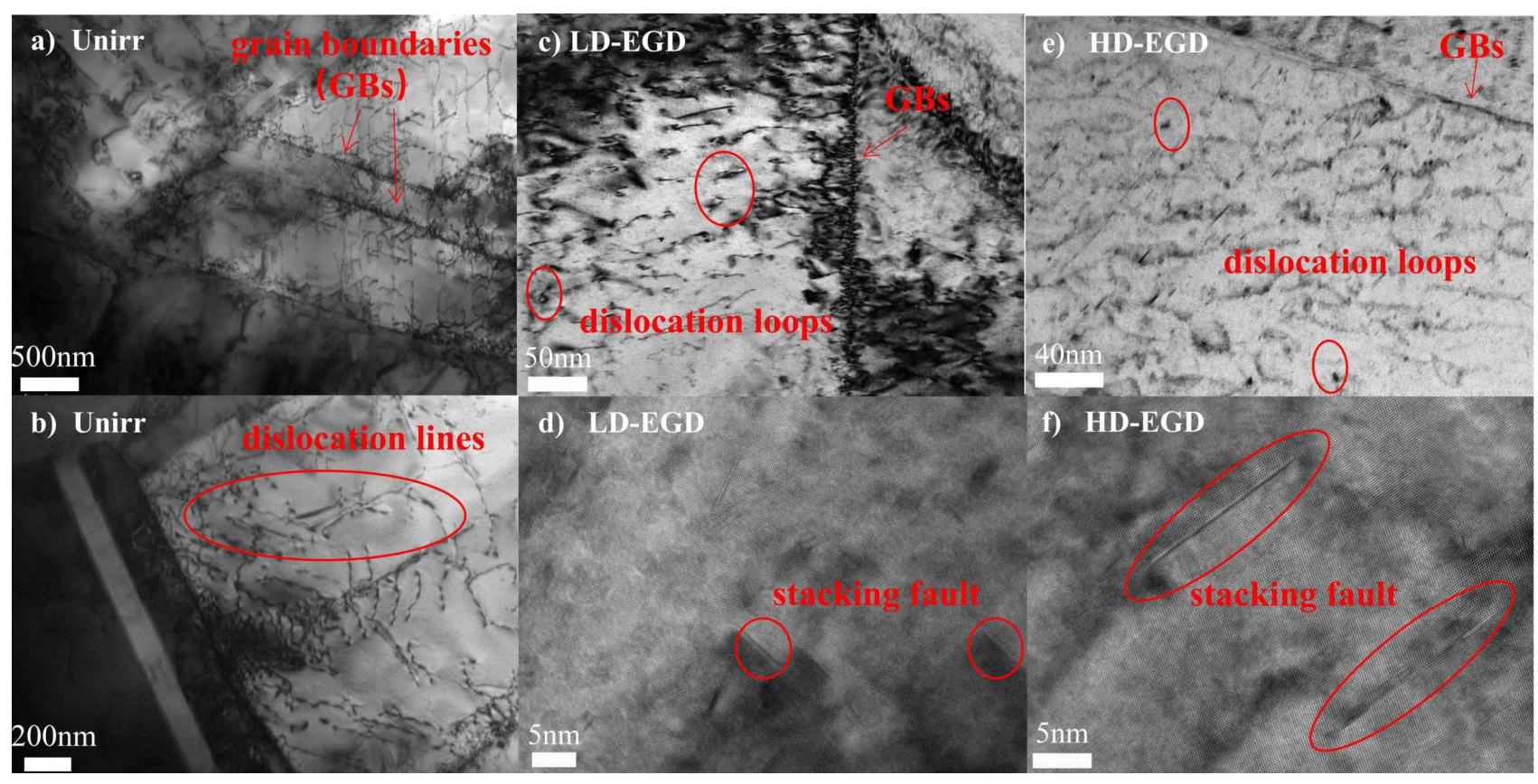

Figure 3. TEM characterization of different samples: (a,b) unirradiated sample, (c,d) low dose with energy gradient degrader (LD-EGD), (e,f) high dose with energy gradient degrader (HD-EGD).
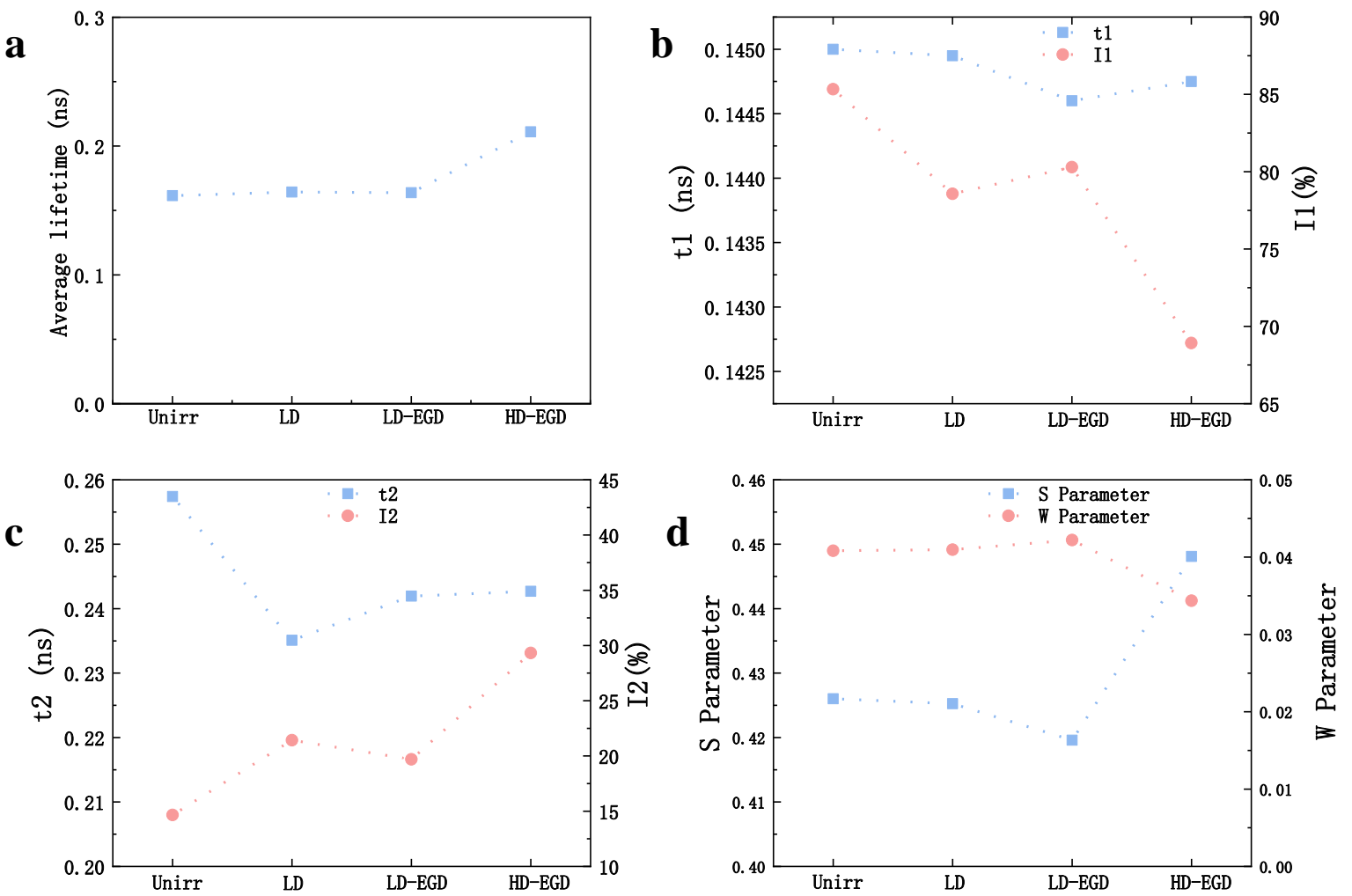

Figure 4. Positron annihilation test results of different irradiated samples: (a) the average lifetime, (b) the lifetime value and intensity of the first life component, (c) the lifetime value and intensity of the second life component, (d) the S parameter and W parameter of the Doppler broadened spectrum. 
According to the previous simulation calculations, the positron annihilation lifetime at the edge dislocation in pure $\mathrm{Ni}$ is about $113 \mathrm{ps}$, the positron annihilation lifetime at the stacking fault tetrahedrons in Ni matrix is between 130 and 183 ps, the positron annihilation lifetime at the single vacancy is about $175 \mathrm{ps}$, and the positron annihilation lifetime of the V4-type microvoids is about 257 ps, respectively [28]. Taking these calculated values as a reference, it is inferred that the first life component mainly reflects the annihilation of the free state and the trapped state at simple defects. Combined with the TEM experimental results, the simple defects mainly include inherent dislocation lines, stacking fault tetrahedron and single vacancies. The second life component is a longer life component, which may mainly characterize the annihilation of microvoids. The third annihilation state presents the characteristics of large lifetime value and extremely low intensity, which mainly reflects the surface information of the sample [29]. Therefore, only the first two life components can reflect the defect information inside the sample. The average values of the lifetime and intensity of the first two life components are further analyzed.

It can be seen from Figure 4a that the average lifetime value of the third irradiated sample HD-EGD has a relatively obvious change, while the average lifetime values of other irradiated samples are not much different from that of the unirradiated sample. This indicates that the irradiation damage effect of the sample HD-EGD is more significant with the appearance of more long-lived defects such as microvoids. As can be seen in Figure $4 b$, the lifetime values change little, and the intensity value shows a decreasing trend after neon ion irradiation. Figure $4 \mathrm{c}$ shows that the lifetime values decrease after irradiation, indicating the reduction in the size of the defects. Meanwhile, it is found that the corresponding annihilation intensity increases after irradiation because more microvoids are formed after the irradiation of neon ions and the probability of capturing positrons increases.

In addition, the Doppler broadened spectrum test is performed on the samples. After linear parameter analysis, the $S$ parameter values and the $W$ parameter values are obtained. The $S$ parameter reflects the information of low-momentum electrons and is more sensitive to vacancy-type defects. The larger the value, the higher the concentration of vacancy-type defects. The $\mathrm{W}$ parameter has the opposite physical meaning [6,29]. Figure $4 \mathrm{~d}$ shows that the two parameter values of the first and second irradiated samples are close to those of the unirradiated sample. For the third irradiated sample HD-EGD, the $S$ parameter value has a relatively obvious increase, while the $\mathrm{W}$ parameter value has a relatively obvious decrease, reflecting the obvious increase in the concentration of vacancy-type defects. The above results can be attributed to the differences in the concentration of inherent defects in the original samples and the irradiation effects. The irradiation damage effects on the first and second irradiated samples are weak, which cannot make up for the difference of the original vacancy-type defect concentration, so the $S$ parameter value is slightly smaller than that of the unirradiated sample. While for the HD-EGD sample, the irradiation effect is more obvious, basically unaffected by the differences in the initial defect concentrations.

\subsection{Mechanical Properties Analysis by Small Punch Test}

\subsubsection{Morphology of Fracture Section after Small Punch Test}

The surface and section of samples are observed with SEM, and the corresponding results are shown in Figures 5 and S3. As is shown in Figure S3, the fractured parts of the samples are not located in the central area but distributed around the periphery. This is in line with the results of the finite element simulation; that is, the place where the stress has the maximum value and the fracture occurs is at some distance from the center of the samples [20]. There is a small raised cap in the middle of the sample. Cracks and small cracks along the radial direction appear on the surface, and the cracks on the sample surface are more obvious after high-temperature annealing. The section morphology shows that a large number of dimples and a small number of ductile tear edges appeared at the sections of both the unirradiated and irradiated samples under different annealing conditions, which shows obvious ductile fracture characteristics (Figure 5a-k) [20]. The 
neon ion irradiation and annealing conditions did not significantly affect the basic fracture characteristics of the alloy.

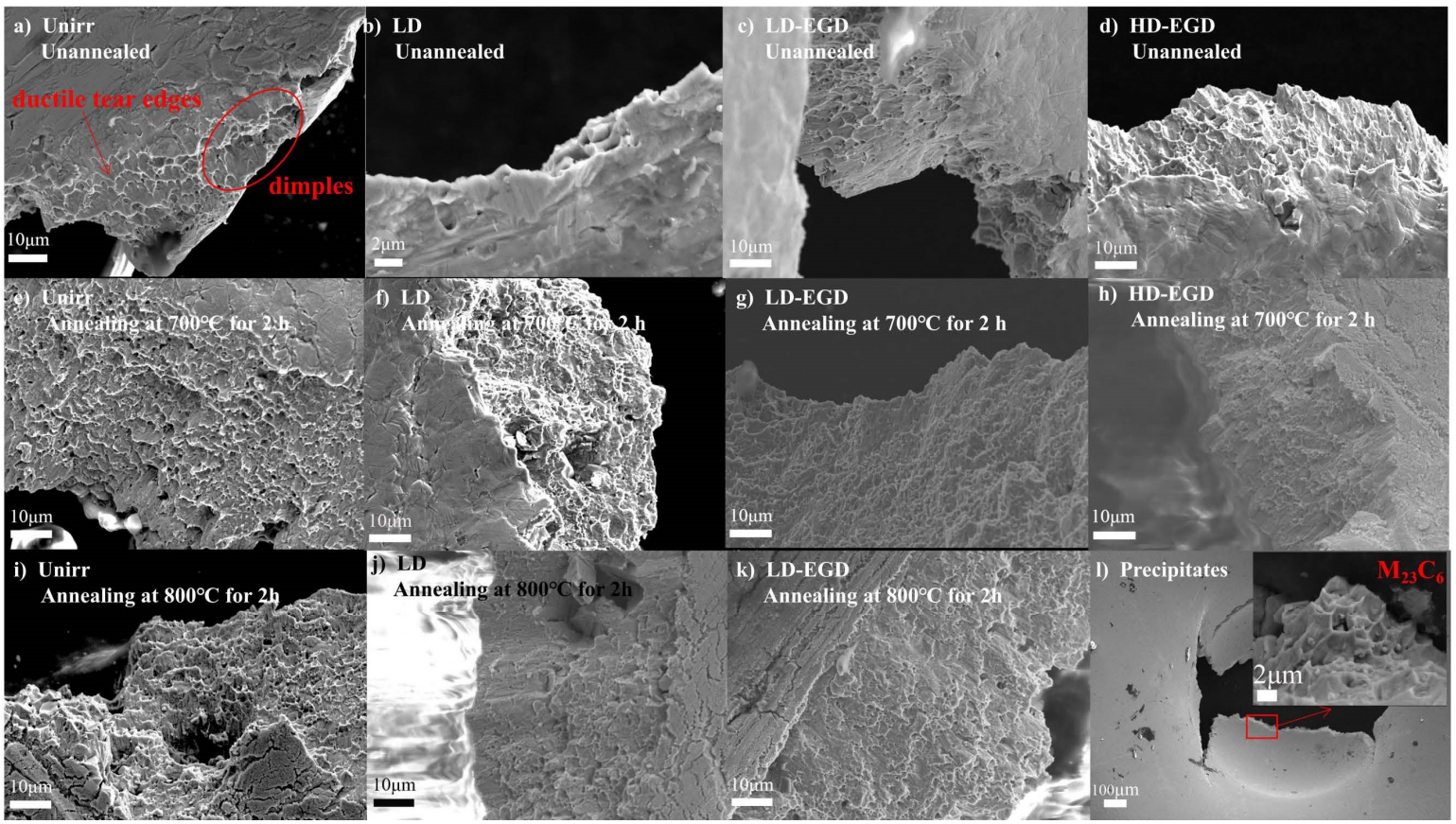

Figure 5. Section morphology of samples under different annealing conditions: (a) unirradiated and unannealed sample, (b) unannealed LD sample, (c) unannealed LD-EGD sample, (d) unannealed HD-EGD sample. (e) Unirradiated and annealed at $700{ }^{\circ} \mathrm{C}$ for $2 \mathrm{~h}$, (f) LD and annealed at $700{ }^{\circ} \mathrm{C}$ for $2 \mathrm{~h}$, (g) LD-EGD and annealed at $700{ }^{\circ} \mathrm{C}$ for $2 \mathrm{~h}$, (h) HD-EGD and annealed at $700{ }^{\circ} \mathrm{C}$ for $2 \mathrm{~h}$. (i) Unirradiated and annealed at $800^{\circ} \mathrm{C}$ for $2 \mathrm{~h},(\mathbf{j}) \mathrm{LD}$ and annealed at $800^{\circ} \mathrm{C}$ for $2 \mathrm{~h},(\mathbf{k}) \mathrm{LD}$-EGD and annealed at $800^{\circ} \mathrm{C}$ for $2 \mathrm{~h}$, (l) precipitates at the fracture section.

In addition, some precipitates are observed in the sample section. Two samples (the second irradiated sample LD-EGD under the unannealed condition and the unirradiated sample annealed at $700{ }^{\circ} \mathrm{C}$ for $2 \mathrm{~h}$ ) are selected, and the point scanning analysis by EDS is performed on the precipitate position of the section and the matrix position without precipitates. The results show that the main components of the precipitates at the cross section are $\mathrm{Cr}$, Mo and $\mathrm{C}$. Studies have shown that $\mathrm{M}_{23} \mathrm{C}_{6}$ carbide is the main precipitate in alloy 617 [30], so it is inferred that the precipitate observed in the experiment is $\mathrm{M}_{23} \mathrm{C}_{6}$ carbide.

\subsubsection{Load-Deflection Curve Analysis}

After the small punch test, the load-deflection curves are obtained and some of the curves are shown in Figure S2. The tensile and fracture characteristic values are obtained from load-deflection curves by using the empirical correlation method. In order to better compare the changes in the mechanical properties of the irradiated samples, the relative tensile characteristic values and the relative fracture characteristic values are calculated based on the unirradiated samples under different annealing conditions in Figure 6.

It can be seen from Figure 6 that almost all the relative tensile characteristic values of the irradiated samples with and without high-temperature annealing are less than 1. The tensile characteristic values of the irradiated samples become smaller, reflecting the decrease in the ultimate tensile strength. It indicates that the irradiation causes the softening behavior of the alloy 617. At present, there are few explanation mechanisms for the irradiation softening effect, mainly from the perspective of the alloy hardening phase. Changizian et al., in their study, indicated that the stability of the $\gamma^{\prime}$ precipitates and the changes of alloy hardness are linked $[17,31]$. The high-energy ion irradiation introduces micro-defects such as dislocation loops but also reduces the stability of the hardening phase 
of alloy 617. The "pinning effect" caused by the micro-defects makes the alloy harden, while the instability of the hardening phase makes the alloy soften. The softening effect is dominant, so the alloy exhibits a softening behavior. After high-temperature annealing, the defects introduced by the irradiation recover, but the hardening phase of the alloy is still unstable, so the irradiated sample still maintains the softening behavior.
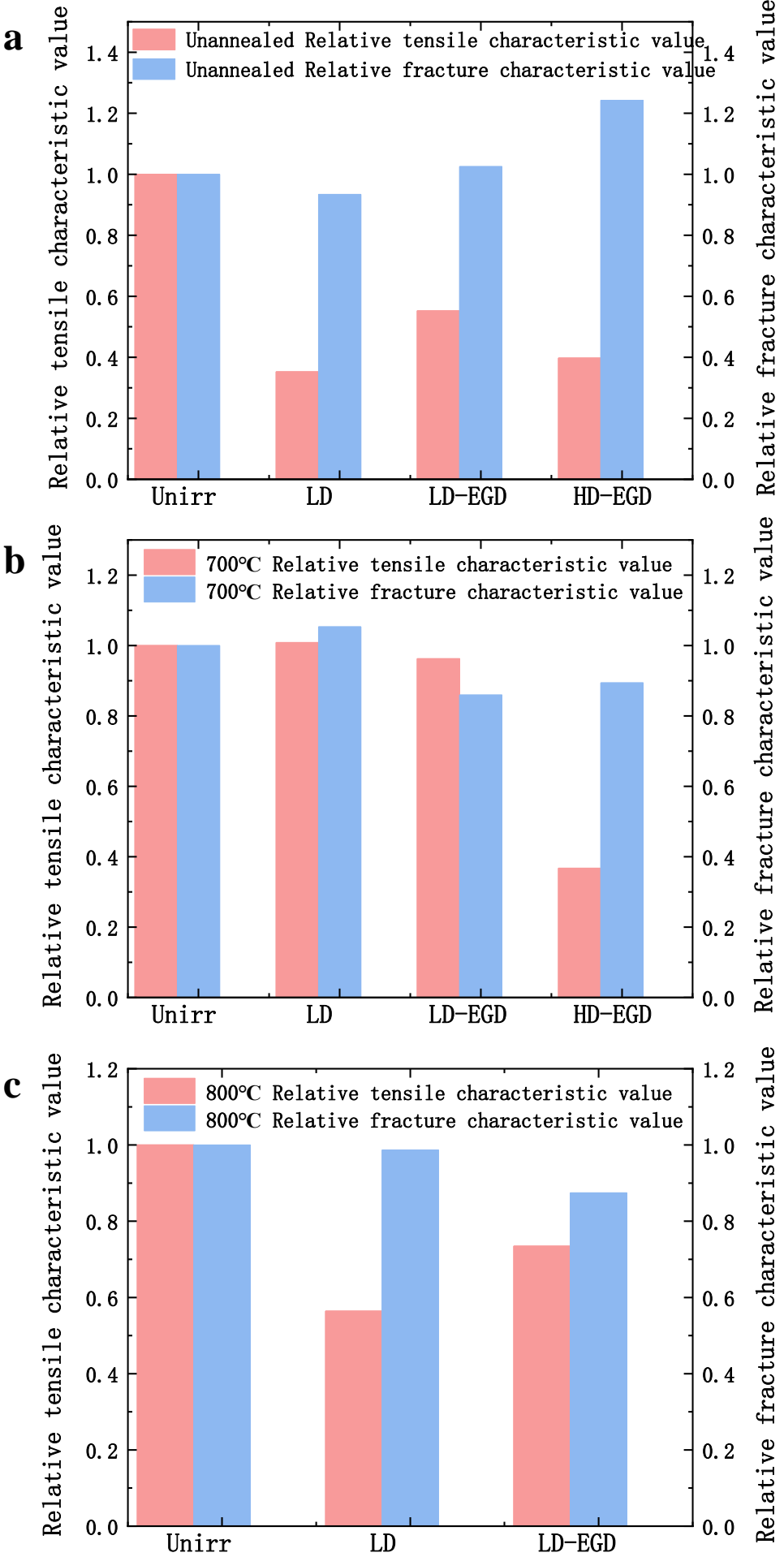

Figure 6. Changes in the characteristic values of tensile strength and fracture toughness of each sample under different annealing conditions:(a) unannealed, (b) annealed at $700{ }^{\circ} \mathrm{C}$ for $2 \mathrm{~h}$, (c) annealed at $800^{\circ} \mathrm{C}$ for $2 \mathrm{~h}$. 
From Figure $6 \mathrm{a}-\mathrm{c}$, it can be seen that the fracture characteristic values of the irradiated sample do not decrease significantly under the unannealed condition, while the fracture characteristic values of the irradiated sample show a more obvious decreasing trend after high-temperature annealing, indicating that the irradiation embrittlement effect is more obvious only after high-temperature annealing. Considering the experimental conditions, the matrix damage caused by neon ion irradiation may be the main factor. From the previous microstructure characterization results, only a few defects were introduced by neon ion irradiation, and the formation of neon bubbles was not observed. During the process of high-temperature annealing, neon ions may migrate and aggregate to form neon bubbles, leading to embrittlement of the alloy. Therefore, the embrittlement effect of the alloy is significantly manifested after high-temperature annealing. This is also in line with the high-temperature embrittlement in the actual reactor.

Moreover, the results partly reflect the applicability of the small punch test for mechanical properties analysis of nickel-based alloys. First, as is shown in Table S1, after the high-temperature annealing, the tensile characteristic value of the unirradiated sample is reduced, indicating a softening phenomenon. It is in line with the general law of the strength change after the high-temperature annealing. This reflects the feasibility of analyzing tensile properties with the tensile characteristic values for nickel-based alloys. Second, it can be seen from Table S1 that the fracture characteristic values of alloy 617 do not change significantly under different annealing conditions, which is similar to the characterization results of SEM. The small changes of the fracture characteristic values reflect the small changes of the fracture toughness after irradiation, so the basic fracture characteristics of the alloy has not changed. These results accord with the previous observations of section morphology. Third, the fracture characteristic value decreases after high-temperature annealing, reflecting the decrease in the fracture toughness. The previous SEM results show that surface cracks are more obvious after annealing, and the fracture toughness is the parameter reflecting the ability of the sample to prevent crack expansion. Therefore, it can be inferred from the SEM results that the fracture toughness decreases after annealing, which is mutually confirmed with the analysis results of the load-deflection curve. To sum up, it can be seen that the small punch test can be used for qualitative analysis of irradiation effects of nickel-based alloys, while quantitative analysis needs further research. Further research will be focused on using the small punch test to evaluate irradiation effects of alloy 617 caused by other ions, so as to explore its applicability for nickel-based alloys.

\section{Conclusions}

Based on the similar migration and aggregation behavior between neon and helium ions, high-energy neon ion irradiation with a maximum energy of $120 \mathrm{MeV}$ is conducted on the nickel-based alloy 617 and the larger atomic displacement cross-section enables it to introduce high displacement damage in the alloy. Meanwhile, in order to make the irradiation damage distribution more even, an energy gradient degrader device is also used for some samples to obtain five incident neon ions with different energies.

(1) No significant difference in the surface morphology is observed between irradiated and unirradiated samples. The neon ion irradiation introduces defects such as dislocation loops and stacking faults and also affects the evolution of defects such as inherent dislocation lines.

(2) The changes in the microstructure after neon ion irradiation causes deterioration of mechanical properties. Under different annealing conditions, the irradiation makes alloy 617 soften and does not lead to changes in its basic fracture characteristics. Alloy 617 still exhibits ductile fracture characteristics, and its fracture toughness tends to decrease after irradiation under high-temperature annealing conditions. Changes in mechanical properties may be related to the instability of the hardened phase and the formation of neon bubbles.

(3) These results could further evaluate and predict the irradiation resistance properties of alloy 617. 
Supplementary Materials: The following supporting information can be downloaded at: https: / / www.mdpi.com/article/10.3390/met12030438/s1, Figure S1: The device schematic of the small punch test; Figure S2: The load-deflection curves of the samples after annealing at $800{ }^{\circ} \mathrm{C}$ for $2 \mathrm{~h}$; Figure S3: $(a, b)$ Overall morphology, $(c-e)$ the cracks on the implanted surface of unirradiated samples under different annealing conditions; Table S1: The tensile characteristic value and fracture characteristic value of unirradiated samples after different annealing treatments.

Author Contributions: Conceptualization, S.L. and L.S. (Libin Sun); methodology, S.L., L.S. (Li Shi) and L.S. (Libin Sun); validation, Z.L.; SRIM calculation, Y.S.; data curation, Y.S. and Q.Z.; writingoriginal draft preparation, Y.S.; writing-reviewing and editing, S.L., Q.Z., L.S. (Li Shi) and Z.L.; supervision, Z.L.; All authors have read and agreed to the published version of the manuscript.

Funding: The project is supported by the Special Funds for the Key Research and Development Program of the Ministry of Science and Technology of China (Grant No. 2020YFB1901800) and the National Natural Science Foundation of China (Grant No. 12005017 and 11975135).

Acknowledgments: This project was supported by the Heavy Ion Accelerator Laboratory (HIRFL), Key Lab of Advanced Materials (MOE), Hubei Key Laboratory of Nuclear Solid State Physics (Wuhan University) and China Institute of Atomic Energy. The irradiation experiment was completed at the Heavy Ion Accelerator Laboratory (HIRFL) of the Lanzhou Institute of Modern Physics. The small punch test was performed at the China Institute of Atomic Energy.

Conflicts of Interest: The authors declare no conflict of interest.

\section{References}

1. Abram, T.; Ion, S. Generation-IV nuclear power: A review of the state of the science. Energy Policy 2008, 36, 4323-4330. [CrossRef]

2. Zinkle, S.J.; Snead, L.L. Designing Radiation Resistance in Materials for Fusion Energy. Annu. Rev. Mater. Res. 2014, 44, 241-267. [CrossRef]

3. World Nuclear News. Alloy Clear for Use in High-Temperature Reactors. 2020. Available online: https://worldnuclear-news.org/Articles / Alloy-qualified-for-use-in-high-temperature-reacto\#: \{\}:text=Alloy $\% 20617 \% 20 \% 2 \mathrm{D} \% 20 \mathrm{a} \% 20$ combination \%20of,Boiler\%20and\%20Pressure\%20Vessel\%20Code.\&text=INL\%20spent $\% 2012 \% 20$ years \%20qualifying, the \%20 US\%20Department $\% 20$ of $\% 20$ Ene (accessed on 29 January 2022).

4. Ren, W.; Swindeman, R.W. A review on current status of alloys 617 and 230 for Gen IV nuclear reactor internals and heat exchangers. J. Press. Vessel Technol. 2009, 131, 044002. [CrossRef]

5. Mo, K.; Lv, W.; Tung, H.M.; Yun, D.; Miao, Y.; Stubbins, J.F. Biaxial Thermal Creep of Alloy 617 and Alloy 230 for VHTR Applications. In Pressure Technology, Proceedings of the ASME 2014 Symposium on Elevated Temperature Application of Materials for Fossil, Nuclear, and Petrochemical Industries, Seattle, WA, USA, 25-27 March 2014; American Society of Mechanical Engineers: New York, NY, USA, 2014; pp. 20-28.

6. Chen, H.C. The Irradiation Effect of Nickel and Nickel-Based Alloy. Ph.D. Thesis, Chinese Academy of Science, Shanghai, China, 2017.

7. Jin, S.X.; He, X.F.; Li, T.C.; Ma, S.; Tang, R.; Guo, L. Microstructural evolution in nickel alloy C-276 after Ar-ion irradiation at elevated temperature. Mater. Charact. 2012, 72, 8-14. [CrossRef]

8. Jin, S.; Guo, L.P.; Ren, Y.Y.; Tang, R.; Qiao, Y. TEM characterization of self-ion irradiation damage in nickel-base alloy C-276 at elevated temperature. J. Mater. Sci. Technol. 2012, 28, 1039-1045. [CrossRef]

9. Kojima, S.; Yoshiie, T.; Kiritani, M. Defect structure evolution from cascade damage in $14 \mathrm{MeV}$ neutron irradiated nickel and nickel alloys. J. Nucl. Mater. 1988, 155, 1249-1253. [CrossRef]

10. Chen, H.C.; Li, D.H.; Lui, R.D.; Huang, H.; Li, J.; Lei, G.; Huang, Q.; Bao, L.; Yan, L.; Zhou, X.; et al. Ion irradiation induced disappearance of dislocations in a nickel-based alloy. Nucl. Instrum. Methods Phys. Res. Sect. B Beam Interact. Mater. At. 2016, 377, 94-98. [CrossRef]

11. Huang, H.F.; Li, D.H.; Li, J.J.; Liu, R.D.; Lei, G.H.; He, S.X.; Huang, Q.; Yan, L. Nanostructure Variations and Their Effects on Mechanical Strength of Ni-17Mo-7Cr Alloy under Xenon Ion Irradiation. Mater. Trans. 2014, 55, 1243-1247. [CrossRef]

12. Huang, H.F.; Li, D.H.; Yan, L. Xenon Ion Irradiation Effects on a Ni-Base Ni-17Mo-7Cr Alloy. Adv. Sci. Technol. 2014, 94, 69-74.

13. Cao, M.P. Study on the Evolution of Irradiation Defect and Irradiation Resistance of Binary Nickel Base Alloys. Master's Thesis, Chinese Academy of Science, Shanghai, China, 2020.

14. Liu, Z. Investigation of helium behavior in Nickel-based alloys of Molten Salt Reactor by using Ion-Beam Technique. Master's Thesis, Chinese Academy of Science, Shanghai, China, 2015.

15. Zhang, S.J.; Li, D.H.; Chen, H.C.; Lei, G.H.; Huang, H.F.; Zhang, W.; Wang, C.B.; Yan, L.; Fu, D.J.; Tang, M. Ion irradiation-induced swelling and hardneing effect of Hastelloy N alloy. J. Nucl. Mater. 2017, 489, 180-186. [CrossRef]

16. Guo, P.; Xue, J.M.; Yan, L.; Huang, Q.; Li, Z.-J.; Huai, P.; Zhou, X.-T. Irradiation Effects in Ni-17Mo-7Cr Alloy Bombarded with $\mathrm{MeV}$ Au Ions. Acta Metall. Sin. Engl. Lett. 2015, 28, 903-908. [CrossRef] 
17. Changizian, P.; Lu, C.; Yao, Z.; Wang, L.M. Indentation behaviour of ion-irradiated X-750 Ni-based superalloy. Philos. Mag. Lett. 2017, 97, 101-109. [CrossRef]

18. Martin, W.R.; Weir, J.R. Effect of Elevated-Temperature Irradiation on Hastelloy N. Nucl. Appl. 1965, 1, 160-167. [CrossRef]

19. Rowcliffe, A.F.; Mansur, L.K.; Hoelzer, D.T.; Nanstad, R.K. Perspectives on radiation effects in nickel-base alloys for applications in advanced reactors. J. Nucl. Mater. 2009, 392, 341-352. [CrossRef]

20. Liu, J.; Zhang, C.; Yang, Y.; Song, Y.; Zhang, L.; Gou, J.; Xian, Y.; Chen, J.; Jang, J. Embrittlement of an ODS Ferritic Steel under High-energy Heavy-ion Irradiation. Nucl. Phys. Rev. 2015, 32, 347-352.

21. Wang, C.; Bai, B.; Zhang, C.; Tong, Z.; Zhong, W.; Xu, S.; Zhao, Y.; Ning, G.; Yang, W. Study on Small Punch Test of China A508-3 Steel Material Irradiated by Fast Neutron at $10 \times 10^{19} \mathrm{~cm}^{-2}$. At. Energy Sci. Technol. 2020, 54, 683-687.

22. Arunkumar, S. Overview of Small Punch Test. Met. Mater. Int. 2020, 26, 719-738. [CrossRef]

23. Torres, J.; Gordon, A.P. Mechanics of the small punch test: A review and qualification of additive manufacturing materials. J. Mater. Sci. 2021, 56, 10707-10744. [CrossRef]

24. Altstadt, E.; Houska, M.; Simonovski, I.; Bruchhausen, M.; Holmström, S.; Lacalle, R. On the estimation of ultimate tensile stress from small punch testing. Int. J. Mech. Sci. 2018, 136, 85-93. [CrossRef]

25. Bruchhausen, M.; Holmström, S.; Simonovski, I.; Austin, T.; Lapetite, J.-M.; Ripplinger, S.; De Haan, F. Recent developments in small punch testing: Tensile properties and DBTT. Theor. Appl. Fract. Mech. 2016, 86, 2-10. [CrossRef]

26. CEN Workshop Agreement. CWA 15627:2007, Small Punch Test Method for Metallic Materials; European Committee for Standardization: Brussels, Belgium, 2007.

27. ASTM. Subcommittee E10.02, 2020, Standard Test Method for Small Punch Testing of Metallic Materials; Version 07/24/20; ASTM: West Conshohocken, PA, USA, 2020.

28. Kuramoto, E.; Tsutsumi, T.; Ueno, K.; Ohmura, M.; Kamimura, Y. Positron lifetime calculations on vacancy clusters and dislocations in Ni and Fe. Comput. Mater. Sci. 1999, 14, 28-35. [CrossRef]

29. Lian, X.Y. The Study of Electron Density Distribution and Positron Annihilation Mechanism of Micro-Defects in Fe-9Cr Alloy. Master's Thesis, East China University of Technology, Jiangxi, China, 2018.

30. Guo, Y.; Hou, S.F.; Zhou, R.C. Effect of Grain-Boundary M23C6 Carbides on Mechanical Properties of Inconel Alloy 617. J. Chin. Soc. Power Eng. 2010, 30, 804-808.

31. Changizian, P.; Brooks, A.; Yao, Z.W.; Daymond, M.R. Nano-scale Mechanical Properties and Microstructure of Irradiated X-750 Ni-Based Superalloy. Metall. Mater. Trans. A 2018, 49, 498-514. [CrossRef] 